\title{
Home for a rest: stem cell niche of the postnatal growth plate
}

\author{
Julian C Lui \\ Section on Growth and Development, Eunice Kennedy Shriver National Institute of Child Health and Human Development, National Institutes of Health, \\ Bethesda, Maryland, USA
}

Correspondence should be addressed to J C Lui: luichunk@mail.nih.gov

\begin{abstract}
The resting zone houses a group of slowly proliferating 'reserve' chondrocytes and has long been speculated to serve as the stem cell niche of the postnatal growth plate. But are these resting chondrocytes bona fide stem cells? Recent technological advances in lineage tracing and next-generation sequencing have finally allowed researchers to answer this question. Several recent studies have also shed light into the signaling pathways and molecular mechanisms involved in the maintenance of resting chondrocytes, thus providing us with important new insights into the role of the resting zone in the paracrine and endocrine regulation of childhood bone growth.
\end{abstract}

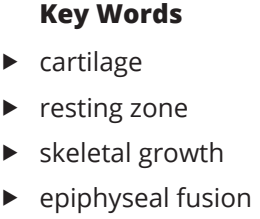

Journal of Endocrinology (2020) 246, R1-R11

\section{Introduction}

Longitudinal bone growth is driven by a process called endochondral ossification, in which growing cartilage is gradually remodeled into newly formed bone (Kronenberg 2003). During embryonic development, this process begins with a condensation of mesenchymal stem cells, which differentiate into type II collagen-producing chondrocytes to form a cartilage mold (Hall \& Miyake 2000). As this cartilage structure gradually expands due to chondrocyte proliferation, at the center of the cartilage, proliferation eventually ceases and hypertrophy begins and the cells differentiate into type $\mathrm{X}$ collagen-producing hypertrophic chondrocytes. These hypertrophic chondrocytes drive cartilage matrix mineralization and eventually undergo apoptosis (Adams \& Shapiro 2002), leaving a scaffold of cartilage matrix for invasion of blood vessels and osteoblasts to lay down bone matrix in the center of the cartilage, which is known as the primary ossification center. Alternatively, recent evidence suggests that these hypertrophic chondrocytes may also directly transdifferentiate into osteoblasts themselves (Yang et al. $2014 a, b$, Zhou et al. 2014). This process of chondrocyte proliferation, followed by hypertrophy and osteoblast invasion, lead to the formation and lengthening of long bones, which drives the increase in body size and length early on (Lui 2017). Shortly after birth, a secondary ossification center starts to form at the epiphysis, dividing the growing cartilage into two cartilage structures of different functions: the articular cartilage, which covers the end of bones to provide a smooth and lubricated surface at the joint; and the epiphyseal growth plate, where endochondral ossification continues to drive bone growth in postnatal life (Fig. 1).

Chondrocytes in the epiphyseal growth plate are arranged into three histologically distinct zones called the resting, proliferative, and hypertrophic zones. Right below the secondary ossification center, chondrocytes in the resting zone are slowly proliferating (Kember 1971) and irregularly scattered in a bed of cartilage matrix. Adjacent to the resting zone, chondrocytes are arranged in columns parallel to the long axis of the bone and within each column, similar to what happens in embryonic development, chondrocytes initially undergo rapid 


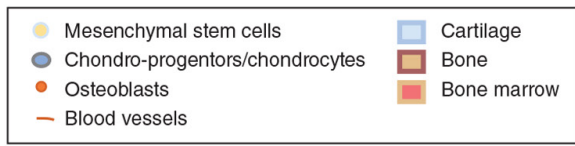

Fetal/neonatal epiphysis Mesenchymal
condensation
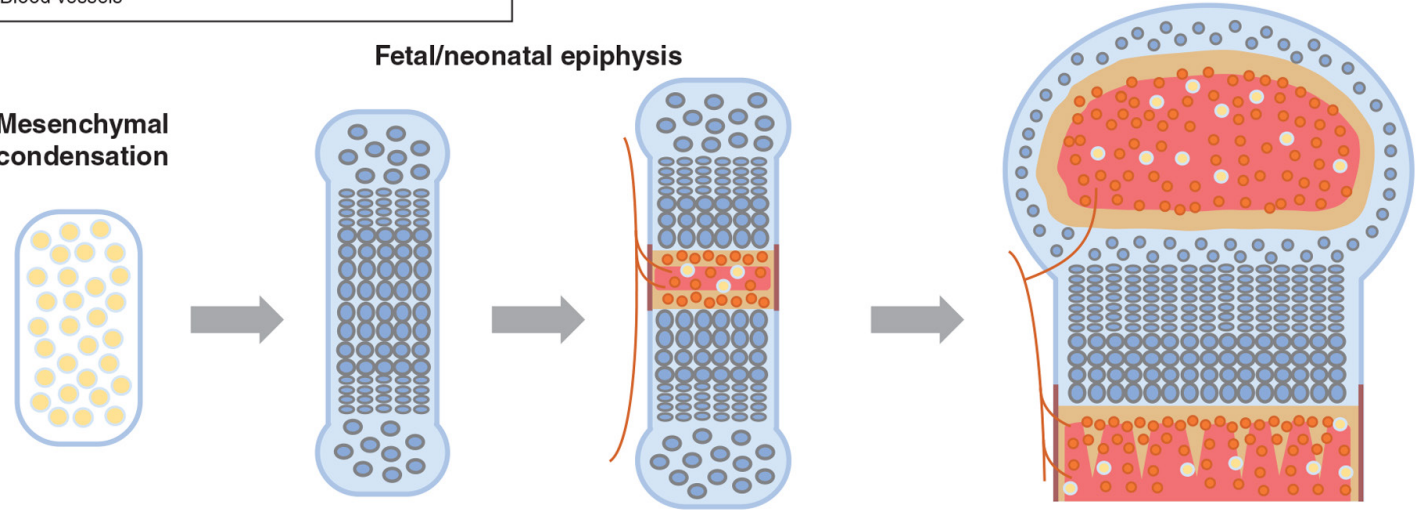

\section{Figure 1}

Endochondral ossification and formation of growth plate. During embryonic development, formation of a mesenchymal condensation is followed by differentiation of mesenchymal stem cells into chondrocytes. The chondrocytes at the center then undergo hypertrophy and apoptosis, drawing the invasion of blood vessels and osteoblasts and building calcified bone in the primary ossification center. Shortly after birth, formation of secondary ossification center separates the epiphysis into articular cartilage and the growth plate, which continues to drive bone elongation.

proliferation, followed by hypertrophy and apoptosis (or transdifferentiation), as incoming blood vessels and osteoblast gradually replace the cartilage matrix with newly formed bone (Hunziker 1994).

\section{Resting chondrocytes: the slowly-cycling 'reserve cells'}

Based on cellular architecture and kinetics, the resting chondrocytes have long been hypothesized to serve as progenitor cells or stem-like cells in the growth plate. They have a much longer cell cycle time than most actively dividing cells (Walker \& Kember 1972, Kember \& Lambert 1981, Ohlsson et al. 1992), which makes them an excellent candidate as 'reserve cells' (Kember 1960) to provide a renewable source to populate the whole growth plate. Direct evidence that resting chondrocytes can give rise to proliferative and hypertrophic columns eventually came in 2002, when Abad et al. surgically excised the proliferative and hypertrophic zone in distal ulnar growth plate in rabbits and showed that the resting zone alone can regenerate a complete growth plate with all three zones (Abad et al. 2002). However, due to several limitations, this study did not unequivocally prove that resting chondrocytes are indeed stem cells of the growth plate. First, this study indicated that resting chondrocytes can differentiate into proliferative chondrocytes after surgical manipulation, but it may not truly reflect the cellular events under normal physiology. Secondly, the precision of the surgical procedure could affect the interpretation of the results. In fact, as the authors admitted, the regeneration of proliferative and hypertrophic columns depends somewhat on the exact location of incision within the resting zone. Thirdly, even if resting chondrocytes indeed give rise to proliferative and hypertrophic chondrocytes in the normal growth plate, it remained possible that the bona fide stem cells are located elsewhere, while resting chondrocytes merely represent one stage of chondrocyte differentiation prior to committing themselves into proliferative columns. For example, if instead only the hypertrophic zone was removed, the proliferative chondrocytes will likely be able to regenerate the hypertrophic columns, but does that mean the proliferative chondrocytes are stem cells? fThere was no direct evidence that these resting zone chondrocytes can form single-cell colonies or differentiate into cells of different lineages. More recent dynamic imaging of the avian embryonic epiphyseal organ culture elegantly demonstrated the in vivo transition of proliferative chondrocyte into the hypertrophic zone, but the recruitment of resting chondrocytes into proliferative columns were harder to be captured and proven definitively (Li et al. 2015).

\section{Lineage tracing provides a powerful tool to reveal cell fates}

Recent advancement in lineage-tracing technology redefined our ability to investigate the fate of a certain 
cell population in vivo without any perturbation. Typically, a transgenic mouse model is generated with cre recombinase driven by a promoter that is only active in a specific cell lineage, such as collagen II for chondrocytes (Hilton et al. 2007). The lineage-specific cre recombinase activity is then used, in many cases activated only upon tamoxifen injection, to permanently label cells with a reporter gene such as yellow fluorescent protein (YFP) (Stadtfeld \& Graf 2005), allowing researchers to follow the fate of a certain cell population and all their descendants both spatially and temporally (Ovchinnikov et al. 2000). There are many clues to what gene promoter can be utilized to specifically label the resting zone. The growth plate is a highly spatially regulated structure with multiple paracrine signaling gradient across the three zones, such as WNTs and BMPs (Lui et al. 2010). In particular, the parathyroid hormone related peptide (PTHrP)-indian hedgehog (IHH) feedback loop is the most well-studied pathway involving the resting zone (Kronenberg et al. 1996). PTHrP is secreted from the perichondrial region in the embryonic cartilage and later from the resting zone after the formation of secondary ossification center (Chen et al. 2006, Chau et al. 2011). PTHrP diffuses down the growth plate, acting on its receptor (PTH/PTHrP receptor) on proliferative chondrocytes to delay the initiation of chondrocyte hypertrophy and production of IHH. At the junction between proliferative and hypertrophic zone, which is sufficiently far away from the resting zone where PTHrP is secreted, IHH is produced. IHH diffuses upward, acting both on its receptor on proliferative chondrocytes to stimulate proliferation and on the resting chondrocytes to stimulate the production of PTHrP (St-Jacques et al. 1999). IHH also acts laterally on the perichondrium to stimulate formation of the bone collar by promoting osteoblast differentiation of the perichondral progenitor cells (Long et al. 2004). The level of PTHrP expression in later postnatal life is not as well-established as earlier in development, but it appears to be an excellent candidate for lineage tracing of resting zone, at least in early life.

\section{Pthrp+ resting chondrocytes behave as skeletal stem cells}

In 2018, Mizuhashi et al. utilized the Pthrp promoter to fluorescent-label a subset of resting zone chondrocytes (thereby referred to as Pthrp-creER+ cells) in embryonic and early postnatal (before postnatal day 7) mouse growth plate (Mizuhashi et al. 2018). Crossing the Pthrp-creER with the Confetti mouse (Livet et al. 2007), which upon cre recombination label each cell and its descendants with one of four fluorescent colors (green, red, yellow, or cyan), revealed that each chondrocyte column was marked by a single unique color, therefore demonstrating that single Pthrp-creER+ ${ }^{+}$resting chondrocytes can clonally give rise to a full column containing both proliferative and hypertrophic chondrocytes. Long-term cell chasing showed that these Pthrp-creER ${ }^{+}$cells continue to form columns within the growth plate for more than a year after the pulse. Descendants of these Pthrp-creER+ cells eventually became Cxcl12-expressing stromal cells and Col1a1-expressing osteoblasts in the trabeculae and primary ossification center, but none differentiated into adipocytes. Colony-forming assay confirmed that, in cultured conditions, these single Pthrp-creER ${ }^{+}$cells can form distinct colonies with more than 50 cells and differentiate into chondrocytes, osteoblasts, and adipocytes upon stimulation (Mizuhashi et al. 2018). Taken together, this elegant study by Mizuhashi et al. provided the most definite proof to date that at least a subset of Pthrp-creER+ resting zone chondrocytes behaves as unipotent stem cells in the growth plate, and with the ability to also become osteoblasts and stromal cells upon transdifferentiation.

\section{Pthrp+ chondrocytes were migrated from the perichondrium}

These Pthrp-creER+ cells did not seem to originate from within the resting zone, but rather came initially from the periphery of the growth plate, immediately adjacent to the perichondrium or an area some referred to as the Ranvier's groove (Fig. 2). Mizuhashi et al. showed using a Pthrp-mCherry reporter mouse, which label active Pthrp-expressing cells rather than marking all decadents arise from Pthrp-creER+ cells, that Pthrp expression was restricted to the periarticular surface and near the Ranvier's groove at birth and appeared to move gradually toward the resting zone in the first week of life (Mizuhashi et al. 2018). By postnatal P9, Pthrp-mCherry+ cells were found primarily at the center of the resting zone and had disappeared from the periphery of the growth plate. This is consistent with our previous finding that the source of Pthrp is gradually shifted from the perichondrial region in the embryonic epiphysis to the resting zone of the postnatal growth plate (Chau et al. 2011). Mizuhashi's study also found that, once the Pthrp-creER+ cells were labeled with tamoxifen injection, they do not expand laterally upon further chase. For instance, when 

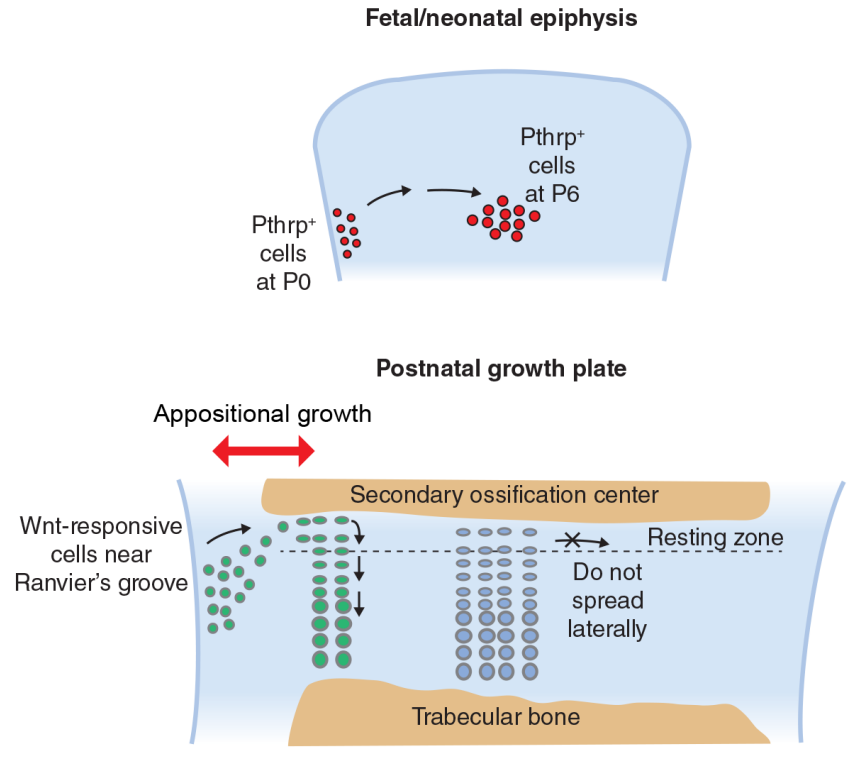

Figure 2

Resting chondrocytes were migrated from perichondrium. Lineage tracing showed that Phtrp-expressing cells gradually migrate from the periphery of the growth plate to the center of the epiphysis during fetal and neonatal period. Later in the postnatal growth plate, cells from the perichondrium near the Ranvier's groove, now labeled with a Wntresponsive Axin2 promoter, continue to migrate to the outer portion of the resting zone, thus contributing to transverse bone growth.

tamoxifen was injected at birth (P0), only a small area of resting zone in the center of the growth plate will be labeled, whether the observation was taken at postnatal P9 or P36. Therefore, it appears that once these PthrpcreER ${ }^{+}$cells have migrated to the resting zone, they do not populate the resting zone laterally but are dedicated for column formation longitudinally.

\section{Wnt-responsive cells contribute to transverse expansion of growth plate}

However, the growth plate also needs to expand transversely. Bone elongation happens when chondrocyte columns are gradually turned into bone. But bone also need to increase in width. It implies that, if Pthrp-creER+ cells do not populate the resting zone laterally, there needs to be another source to contribute to the stem cell pool in the resting zone postnatally as the growth plate gradually expand in width. One possibility is that cells continue to migrate from areas near the perichondrium into the resting zone. However, as shown by the Pthrp$m$ Cherry mouse, Pthrp expression was largely absent near the perichondrium postnatally, so even if it was true that cells from the perichondrium continue to replenish the resting zone, they will no longer be labeled by Pthrp-creER.

(C) 2020 Society for Endocrinology Published by Bioscientifica Ltd. Printed in Great Britain
Recent work by Usami et al. seem to have confirmed the postnatal source of new resting zone chondrocytes. They successfully fluorescent-labeled a group of Wnt/ $\beta$-catenin signaling responsive cells using the Axin-related protein (Axin2) promoter (Axin2-creER+, thereby referred to as Wntresponsive cells) (Usami et al. 2019). When tamoxifen was injected at postnatal P6, these Wnt-responsive cells were found three days later (P9) to reside at the periphery of the growth plate facing the Ranvier's groove, similar to where the Pthrp-creER+ cells were originated. These Wntresponsive cells are slow-cycling and display cell surface markers of osteo-and chondro-progenitor cells (Chan et al. 2018). Extended cell chasing showed that these Wntresponsive cells gradually expanded from the periphery of the growth plate and constituted the outer portion of the growth plate over time, including the outer part of the resting zone (Fig. 2). The timing of their expansion coincides with the transverse appositional growth of the growth plate. When tamoxifen was injected at P21, the expansion of Wnt-responsive cells was promptly observed at P24, but this process was much slower when tamoxifen was injected at P42 or P70, when both transverse and longitudinal growth of the growth plate had substantially decelerated. Interestingly, when injected at P21 or P42, some resting chondrocytes near the center of the growth plate were also labeled after 3 days. These Wnt-responsive cells were unlikely to have migrated from the periphery due to the short chase time, thus suggesting that some of the resting chondrocytes are responsive to Wnt signaling. Taken together, Usami's study suggests that these Wntresponsive cells from the periphery of the growth plate provided a source of resting zone chondrocytes as the growth plate expands transversely in postnatal life. Nevertheless, whether or not these Wnt-responsive cells also express Pthrp as they move into the resting zone is unclear.

\section{Resting zone: a stem cell niche in the epiphyseal growth plate}

In 2019, Newton et al. crossed the Col2a1-creER with the Confetti mouse to study growth plate dynamics (Newton et al. 2019). Unlike the Pthrp-creER or the Axin2-creER, the Col2a1-creER labels all chondrocytes upon tamoxifen injection, but because the Confetti will assign each cell and its descendants with one of four possible colors, this system is well-suited for study of clonality in long-term chase. Interestingly, they observed that many clones (chondrocytes with the same color) formed during the 
fetal and neonatal period were only a few cells long in length and that the clonal size increased considerably after the formation of secondary ossification center, such that all chondrocytes in a single column were marked with the same color in a 1-month-old mouse (Fig. 3). The authors from this study explained that their observations indicated that some chondroprogenitors were slowly depleted by direct recruitment during fetalneonatal growth. In other words, in the progenitor cells that sit in the area designated for the future resting zone, as they proliferate to form chondrocyte columns, some of them were also being recruited into the same column that they produced, moving down and out of the resting zone. Their findings helped explain previous quantitative measurements showing a dramatic reduction of resting zone size in the first 2 weeks of postnatal life in mice (Lui et al. 2018) and a tendency to find clusters of short columns that does not span through the whole height of the growth plate during this period (Fig. 3). These authors also reasoned that their findings suggested that, after the formation of the secondary ossification center, these chondroprogenitors acquired the capacity for self-renewal, thus capable of forming longer columns. Evidently, delaying the formation of secondary ossification center by angiogenesis inhibitor axitinib also delayed the ability of these resting chondrocytes to form monoclonal columns. Therefore, they concluded that their findings indicated the development of a stem cell niche in the postnatal epiphyseal growth plate, allowing a 'radical switch' in clonality, which coincides temporally with the formation of secondary ossification center.

While their study, together with Mizuhashi's recent work (Mizuhashietal.2018), provided convincing evidence that the resting zone is a stem cell niche in the postnatal growth plate, questions remain as to whether there is a truly fundamental 'switch' in these resting chondrocytes to acquire the ability to self-renew and form bigger clones. As stated by Newton et al., many chondroprogenitor cells in the resting zone were depleted by direct recruitment into the proliferative zone. One possible explanation is that these chondroprogenitor cells early on did not yet behave like stem cells and are not capable of self-renewal and that a 'switch' happens around 3-weeks of age, which could be cell-autonomous or triggered by changes in the microenvironment (upon the establishment of the secondary ossification center and the stem cell niche). A second possibility is that there could be more than one population of chondroprogenitor cells in the resting zone. One could imagine that the chondroprogenitor cells sitting right above the proliferative zone may not be true stem cells and were being recruited into columns during the neonatal period, while another population of chondroprogenitor cells more distal from the proliferative zone might always have the capacity to self-renew but remained quiescent during this time, only being 'switched on' after the formation of secondary ossification center.
A Fetal/neonatal epiphysis

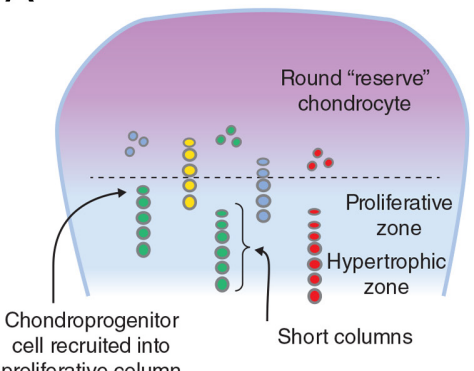
proliferative column

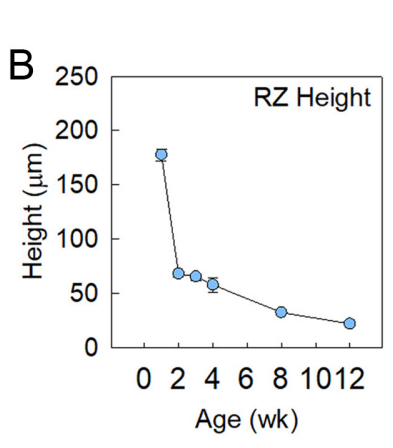

Postnatal growth plate

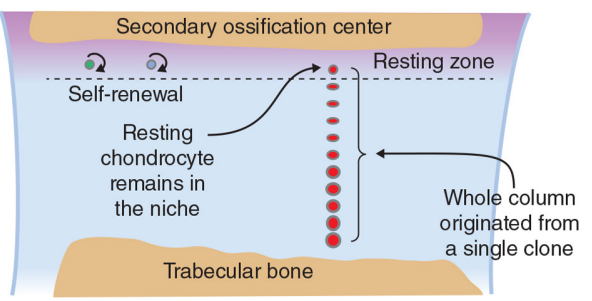

2wk

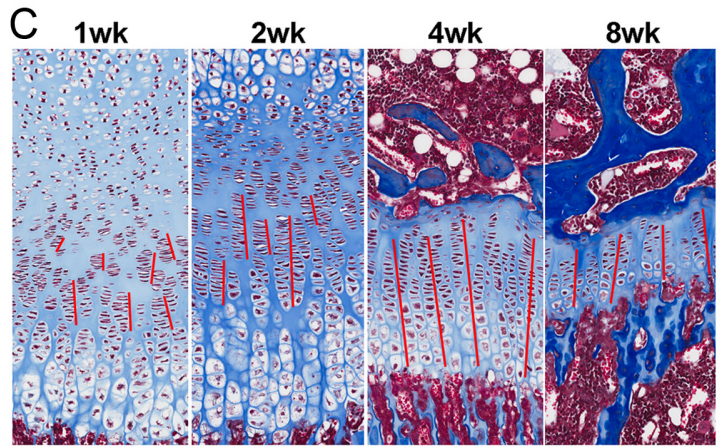

Figure 3

Establishment of resting zone stem cell niche. In fetal and neonatal life, some chondroprogenitor cells in the epiphysis are recruited into the proliferative columns, leading to their gradual depletion early on. However, after the formation of secondary ossification center, the balance appears to shift toward resting chondrocyte self-renewal, leading to formation of long columns from single clones (A). This is consistent with observations that resting zone height in tibia decreases drastically after 1 week in mice (B) and the tendency to find clusters of short columns in growth plate histology of 1- and 2-week-old mice, which gradually transition to more continuous straight columns in older animals, as indicated by the red lines $(C)$. 
A third possibility is that all resting zone chondrocytes may be capable of self-renewing as long as they are in the right microenvironment or receiving the right signals, as it was shown for stem/progenitor cells in other organs (Morrison \& Spradling 2008, Barker et al. 2010, Hsu et al. 2014). During the neonatal period, the chondroprogenitor cells in the resting zone might be more 'prone' to being recruited into the proliferative zone if there is more robust IHH signaling, which can stimulate resting chondrocyte to differentiate into columnar chondrocytes (Kobayashi et al. 2005). The transition from chondroprogenitor depletion to self-renewal could be the result of a gradual change in paracrine signal(s) in the growth plate microenvironment (Fig. 4).

Similarly, the ability to form longer columns could well be a 'switch' that happens in resting chondrocytes after the formation of the stem cell niche. However, it is worth pointing out that the transition from 'chondroprogenitor depletion' to 'chondroprogenitor renewal' itself could lead to formation of longer columns, without postulating a fundamental 'switch' in clonality of chodroprogenitors.
If in an older animal resting chondrocyte depletion is infrequent, the topmost flat chondrocyte differentiated from resting chondrocyte can remain as 'matriarch' of a column for a long time, generating a long column of cells. But in a younger animal, when a chondroprogenitor cell that is making column is being moved down and out of the resting zone (depleted), another chondroprogenitor cells above it may start to make a new column, resulting in two columns (of different colors if using the Confetti) one on top of another. Therefore, the recruitment of chondroprogenitor cells away from the resting zone will inevitably lead to the observed formation of shorter columns, just because the 'matriarch' of a column is swept down toward the hypertrophic zone. It is yet unknown that, if a resting chondrocyte had been allowed to stay in the resting zone and not being recruited, whether or not formation of long column is possible. Therefore, current findings did not unequivocally prove that a 'switch' must exist in the resting chondrocytes to acquire the ability to self-renew and form bigger clones but raised some interesting question for future studies.

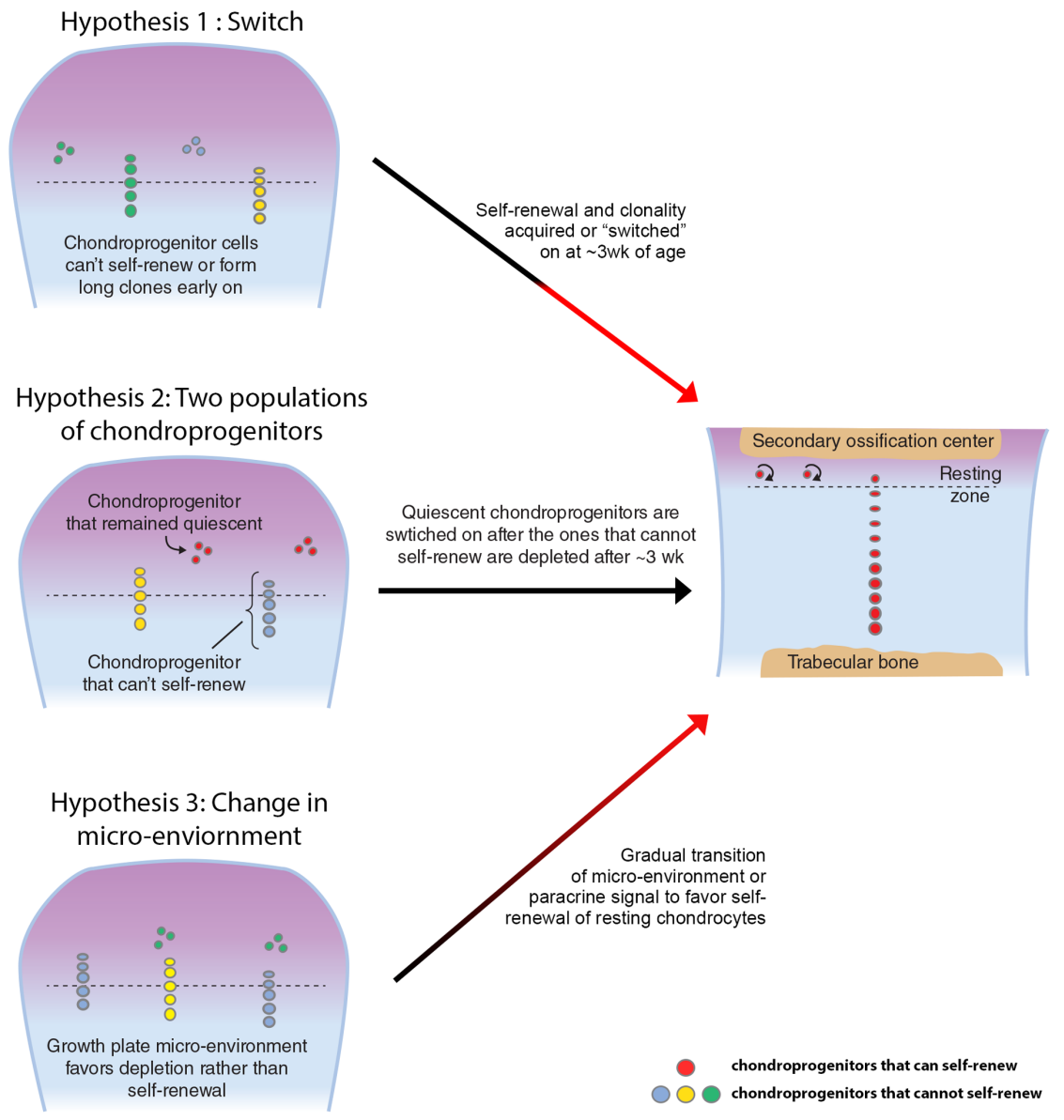

\section{Figure 4}

Alternative hypotheses to explain how resting chondrocytes acquire the ability to self-renew. Hypothesis 1: As described by Newton et al., chondroprogenitor cells may undergo a radical switch at around 3 weeks of age to acquire self-renewal capacity and increased clonality. Hypothesis 2: There may be two distinct populations of chondroprogenitors, one that do not self-renew and get depleted in the first few weeks of life, after which the bona fide stem cells with self-renewal capacity get switched on. Hypothesis 3: Change of microenvironment (such as paracrine signal(s)) in the epiphysis results in a gradual transition of resting chondrocytes from favoring depletion to favoring self-renewal. 


\section{Resting chondrocyte depletion and growth cessation}

In humans, overall longitudinal bone growth happens rapidly during fetal life and early childhood but progressively slows to a halt by late adolescence. Toward the end of bone growth, as least in humans, the growth potential is exhausted and growth plate fusion occurs. Similar declines in bone growth rates also happen in other mammals. This growth deceleration is caused primarily by a decrease in chondrocyte proliferation and hypertrophy, other structural changes in the growth plate, as well as changes in molecular pathways, such as decrease in IGF and WNT signaling with age (Lui et al. 2018), which we collectively termed growth plate senescence (Lui et al. 2011). Interestingly, growth plate senescence also appears to progress at different rates in different long bones in our body, such that these structural and functional senescent changes occur earlier in the growth plates of smaller bones, like the phalanges of our fingers, than in the growth plates of larger bones, such as the tibias. Mother nature appears to modulate growth plate senescence in different bones to achieve the striking disparities in final bone length within a species (Lui et al. 2018). Could growth plate senescence and cessation of growth be driven primarily by the depletion of resting chondrocyte and/or deterioration of the stem cell niche with age?

There are several aspects in testing this hypothesis. First, does depletion of resting chondrocytes lead to premature cessation of growth? Mizuhashi et al. showed that partially ablating Pthrp-expressing cells in the resting zone with diphtheria toxin (Pthrp-creER;R26DTA) significantly reduced the height of proliferative zone, causing premature hypertrophic differentiation (Mizuhashi et al. 2018); however, without testing its effect on growth cessation. This observation is perhaps expected because PTHrP is involved in suppressing chondrocyte hypertrophy in feedback loop with IHH and therefore any experimental model trying to deplete the resting zone will inevitably also disrupt the PTHrPIHH feedback loop. This signifies the challenge in any model attempting to distinguish the dual role of resting chondrocyte, one being the stem cell pool and the other being the source of PTHrP. In theory, one could try to ablate the resting chondrocytes with diphtheria toxin (Voehringer et al. 2008) in a mouse model where chondrocyte hypertrophy is not dependent on PTHrP levels, such as mice with constitutively active PTH/PTHrP receptor (Schipani et al. 1997). However, such a model is not without limitations. These mice with constitutively active PTH/PTHrP receptor have delayed chondrocyte hypertrophy and bone mineralization, and so to assess the effect of resting zone ablation in such model could be complicated. In addition, the ablation of target cells by diphtheria toxin is almost always incomplete, and diphtheria toxin could be released by the ablated cells, causing unintended ablation of surrounding 'non-target' cells.

Secondly, are there quantitative and qualitative changes to the resting zone with age? Indeed, earlier work showed that resting zone height and cell count gradually decline with age in rabbits (Schrier et al. 2006) and, more recently, in rodents (Lui et al. 2018). Interestingly, when growth-inhibiting conditions such as glucocorticoid excess and hypothyroidism temporarily slow growth, they also delayed the overall growth plate senescence, including the decline in resting zone height and cell count, suggesting that resting chondrocytes might be gradually exhausted as one gets more growth out of them (Forcinito et al. 2011). Previous studies on changes of gene expression of the growth plate had largely relied on techniques such as laser capture microdissection (LCM) to separate different zones based on cell morphology (Wang et al. 2004), which is more challenging for the resting zone, until the recent advancement in lineage tracing and single-cell sequencing. Newton et al. recently combined LCM with single-cell RNA Seq to compare the expression of chondroprogenitor cells at postnatal P2 and P28 (Newton et al. 2019) and identified differences in several pathways including extracellular matrix, Wnt signaling, and ERK1/2 signaling. It would be really exciting to apply the same approach to compare the expression profile of resting zone chondrocytes from later postnatal time points, albeit perhaps technically challenging, when bone elongation gradually slows with age. Importantly, with increasing age, the height of the proliferative column gradually decreases; could that be due to a gradual decline of PTHrP signaling from the resting zone, which determines where hypertrophy starts? Does PTHrP expression in all resting chondrocytes gradually decline with age or does the proportion of Pthrpexpressing cells within the resting zone gradually decrease with age? It would also be informative to compare the colony-forming ability of resting zone chondrocytes isolated from growth plate at different ages, perhaps by isolating Pthrp-mCherry+ cells with fluorescence activated cell sorting (FACS), to see if there is any temporal decline in stem cell functionality. 


\section{Estrogen signaling accelerates skeletal maturation}

Estrogen exposure is well-known to positively regulate skeletal maturation and growth plate fusion (Chagin \& Savendahl 2007). Patients with precocious puberty, thus early exposure to estrogen, have accelerated skeletal maturation, early epiphyseal fusion, and consequently decreased final height (Nilsson et al. 2005). Conversely, lack of estrogen due to hypogonadism results in delayed skeletal maturation and increased final height. Remarkably, in the absence of estrogen or estrogen receptor- $\alpha$ (ER- $\alpha)$ (Smith et al. 1994, 2008, Morishima et al. 1995), the growth plate virtually do not fuse at all. Does estrogen signaling drive resting zone chondrocyte depletion to accelerate skeletal maturation? Nilsson et al. showed that injection of estrogen in juvenile ovariectomized rabbits accelerated the normal decline in growth plate height that happened with age (Nilsson et al. 2014). Importantly, estrogen injection also accelerated the loss of resting zone chondrocytes with age, which was not reversed even after discontinuation of estrogen treatment, suggesting a permanent loss of growth potential. The effect of estrogen on chondrocytes is likely mediated by estrogen signaling through ER- $\alpha$, as suggested by the human phenotype (Smith et al. 1994) and further supported by comparing the effects of ER- $\alpha$ and ER- $\beta$ agonist on mouse bone growth (Iravani et al. 2017). Although, whether or not these observed effects were specifically directed toward resting zone chondrocytes remained unclear.

\section{Other signaling pathways important for the resting zone niche}

In addition to estrogen signaling, there are recent evidence suggesting that other signal pathways, such as hedgehog signaling, may also affect the maintenance of resting zone stem cell niche (Fig. 5). Back in 2005, Kobayashi et al. generated a mouse model with mosaic ablation of PTH receptor in growth plate chondrocytes (Kobayashi et al. 2005). Due to the lack of PTHrP signaling, these chondrocytes, some of which are high up in the periarticular region (the future resting zone) of the fetal epiphyseal cartilage, started producing IHH in the region. Interestingly, the elevated level of IHH stimulated the proliferation of round (or resting) chondrocytes and, as the authors suggested, differentiated into columnar (or proliferative) chondrocytes. Curiously, recent studies from Mizuhashi et al. and Newton et al. both examined the

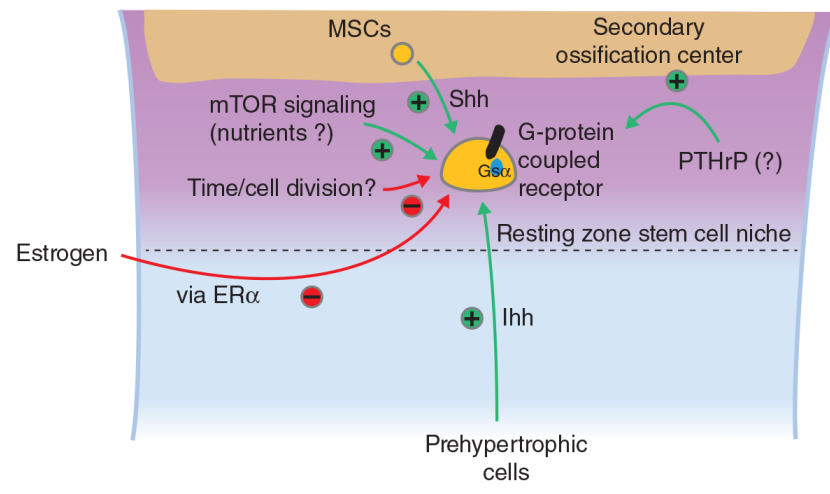

Figure 5

Signaling pathways and factors regulating the maintenance of resting zone chondrocyte in the niche. +, stimulation or positive effector; -, inhibition or negative effector.

effect activation of hedgehog signaling by administration of Smo agonist SAG on resting zone chondrocytes. In Mizhuhashi's study, where SAG was administered at postnatal P6, positive effect on resting chondrocyte proliferation was not observed. However, in Newton's study, where SAG was administered at postnatal P31-34, increase in resting zone chondrocyte proliferation was observed (Newton et al. 2019), consistent with Kobayashi's previous findings. It is yet unclear if the dichotomous outcome on resting chondrocyte in the two studies could be due to stage-specific and contextdependent roles of hedgehog signaling in the stem cell niche. Importantly, although IHH is normally produced in the prehypertrophic zone, which is distant from the resting zone, Newton et al. also found abundant Sonic hedgehog $(\mathrm{SHH})$ expression by mesenchymal stem cells in the secondary ossification center, which is much closer to the resting zone. They have therefore possibly identified the physiological source of hedgehog ligand for the resting chondrocytes and perhaps also explained why the presence of a secondary ossification center is crucial for maintaining a functional resting zone.

Recent studies suggested that another signaling pathway(s) important for stem cell function involve G-protein-coupled receptors (GPCRs). GPCR is a family of receptors with seven-transmembrane domain and mediate their signaling through heterotrimeric G-protein complexes composed of alpha, beta, and gamma subunits. In particular, the stimulatory $G$ alpha subunit $\left(G \alpha_{s}\right)$, which activates adenylate cyclase to catalyze synthesis of cyclic AMP from ATP, is important for skeletal growth. Mutations of GNAS1 (the gene that encodes $G \alpha_{s}$ ) in humans lead to Albright's hereditary osteodystrophy with short stature, brachydactyly, and other skeletal defects (Weinstein et al. 
1992). Interestingly, cartilage-specific Gnas 1 deletion led to severe epiphyseal and growth plate defects with shortening of the proliferative zone and accelerated hypertrophic differentiation of growth plate chondrocytes (Sakamoto et al. 2005), a phenotype strikingly similar to that of the PTH receptor knockout (Lanske et al. 1999). Importantly, more detailed comparison between mouse knockout models of Gs $\alpha$ and PTH receptor showed that ablation of the Gs $\alpha$ alone resulted in accelerated recruitment of resting chondrocytes into proliferative columns, while PTH receptor knockout, or ablation of Gs $\alpha$ combined with a mutant PTH receptor that cannot activate $\mathrm{G}_{\mathrm{q} / 11} \alpha$ (which activates PLC $\beta$ rather than adenylate cyclase), resulted in a more severe phenotype with also increased apoptosis in the resting zone (Chagin et al. 2014). Taken together, these findings suggested that $G s \alpha$ and $G_{q / 11} \alpha$ worked together to preserve the quiescence state and survival of resting zone chondrocytes. Many GPCRs are expressed in chondrocytes and could activate Gs $\alpha$. PTH receptor only appears to be the one responsible for activating Gs $\alpha$ based on the obvious role of PHTrP in resting zone and the aforementioned phenotypic comparisons. Therefore, involvements of other GPCRs, such as the G proteincoupled estrogen receptor 1 (GPER1) (Iravani et al. 2019), should be carefully considered as well.

Another signaling pathway recently linked to resting chondrocyte functions involves the mammalian target of rapamycin (mTOR). mTOR is a nutrient-sensing protein kinase that regulates numerous cellular processes, such as cell growth and proliferation, protein synthesis, and cell autophagy. Earlier studies showed that inhibition of mTOR using rapamycin in fetal metatarsals suppressed hypertrophy (Phornphutkul et al. 2008). Similarly, pharmacological activation of mTOR complex 1 (mTORC1) promotes bone growth in organ culture system (Newton et al. 2015). Furthermore, disruption of mTOR complex 1 (mTORC1) signaling by deletion of mTOR or the associated protein Raptor significantly diminished embryonic skeletal growth with decreased cell size and delayed hypertrophy (Chen \& Long 2014, Jiang et al. 2017). These studies collectively suggest that mTORC1 signaling is growthpromoting and acts primarily by supporting hypertrophy and cell enlargement, which is consistent with the role of mTOR as a nutrient-sensor. More recent studies have shown that mTORC1 signaling might also be involved in the maintenance of the resting zone. Hyperactivation of mTORC1 by ablation of Tuberous sclerosis 1 (TSC1, which normally inhibits mTORC1 activity) in the postnatal growth plate led to disorganization in the resting zone (Newton et al. 2018), which was apparently caused by a subtle shift from asymmetric to symmetric cell division in resting chondrocytes without significant increase in proliferation rate (Newton et al. 2019). More studies will be needed to confirm this finding and investigate how nutritional status might affect the balance of mTOR signaling in cartilage.

\section{Concluding remarks}

Thanks to the recent technological advances, we learned more about the resting zone in the past 2 years than we have in the last two decades. But so much more is yet to be unveiled. How does the resting chondrocyte 'know' when growth potential runs out? How do different endocrine signals and nutritional status regulate the maintenance of resting chondrocytes? This exciting era of stem cell research in skeletal biology holds much promise of providing further insights into childhood bone growth under physiological and pathological conditions.

\section{Declaration of interest}

The author declares that there is no conflict of interest that could be perceived as prejudicing the impartiality of this review.

\section{Funding}

This work was supported by the Intramural Research Program of the Eunice Kennedy Shriver National Institute of Child Health and Human Development, $\mathrm{NIH}$.

\section{References}

Abad V, Meyers JL, Weise M, Gafni RI, Barnes KM, Nilsson O, Bacher JD \& Baron J 2002 The role of the resting zone in growth plate chondrogenesis. Endocrinology 143 1851-1857. (https://doi. org/10.1210/endo.143.5.8776)

Adams CS \& Shapiro IM 2002 The fate of the terminally differentiated chondrocyte: evidence for microenvironmental regulation of chondrocyte apoptosis. Critical Reviews in Oral Biology and Medicine 13 465-473. (https://doi.org/10.1177/154411130201300604)

Barker N, Bartfeld S \& Clevers H 2010 Tissue-resident adult stem cell populations of rapidly self-renewing organs. Cell Stem Cell 7 656-670. (https://doi.org/10.1016/j.stem.2010.11.016)

Chagin AS \& Savendahl L 2007 Estrogens and growth: review. Pediatric Endocrinology Reviews 4 329-334.

Chagin AS, Vuppalapati KK, Kobayashi T, Guo J, Hirai T, Chen M, Offermanns S, Weinstein LS \& Kronenberg HM 2014 G-protein stimulatory subunit alpha and Gq/11alpha G-proteins are both required to maintain quiescent stem-like chondrocytes. Nature Communications 5 3673. (https://doi.org/10.1038/ncomms4673)

Chan CKF, Gulati GS, Sinha R, Tompkins JV, Lopez M, Carter AC Ransom RC, Reinisch A, Wearda T, Murphy M, et al. 2018 Identification of the human skeletal stem cell. Cell 175 43.e21-56. e21. (https://doi.org/10.1016/j.cell.2018.07.029) 
Chau M, Forcinito P, Andrade AC, Hegde A, Ahn S, Lui JC, Baron J \& Nilsson O 2011 Organization of the Indian hedgehog - parathyroid hormone-related protein system in the postnatal growth plate. Journal of Molecular Endocrinology 47 99-107. (https://doi.org/10.1530/JME10-0177)

Chen J \& Long F 2014 mTORC1 signaling controls mammalian skeletal growth through stimulation of protein synthesis. Development 141 2848-2854. (https://doi.org/10.1242/dev.108811)

Chen X, Macica CM, Dreyer BE, Hammond VE, Hens JR, Philbrick WM \& Broadus AE 2006 Initial characterization of PTH-related protein gene-driven lacZ expression in the mouse. Journal of Bone and Mineral Research 21 113-123. (https://doi.org/10.1359/JBMR.051005)

Forcinito P, Andrade AC, Finkielstain GP, Baron J, Nilsson O \& Lui JC 2011 Growth-inhibiting conditions slow growth plate senescence. Journal of Endocrinology 208 59-67. (https://doi.org/10.1677/ JOE-10-0302)

Hall BK \& Miyake T 2000 All for one and one for all: condensations and the initiation of skeletal development. BioEssays 22 138-147. (https://doi.org/10.1002/(SICI)1521-1878(200002)22:2<138:: AID-BIES5 $>3.0 . C O ; 2-4)$

Hilton MJ, Tu X \& Long F 2007 Tamoxifen-inducible gene deletion reveals a distinct cell type associated with trabecular bone, and direct regulation of PTHrP expression and chondrocyte morphology by Ihh in growth region cartilage. Developmental Biology 308 93-105. (https://doi.org/10.1016/j.ydbio.2007.05.011)

Hsu YC, Li L \& Fuchs E 2014 Emerging interactions between skin stem cells and their niches. Nature Medicine 20 847-856. (https://doi. org/10.1038/nm.3643)

Hunziker EB 1994 Mechanism of longitudinal bone growth and its regulation by growth plate chondrocytes. Microscopy Research and Technique 28 505-519. (https://doi.org/10.1002/jemt.1070280606)

Iravani M, Lagerquist M, Ohlsson C \& Savendahl L 2017 Regulation of bone growth via ligand-specific activation of estrogen receptor alpha. Journal of Endocrinology 232 403-410. (https://doi.org/10.1530/JOE16-0263)

Iravani M, Lagerquist MK, Karimian E, Chagin AS, Ohlsson C \& Savendahl L 2019 Effects of the selective GPER-1 agonist G1 on bone growth. Endocrine Connections 8 1302-1309. (https://doi.org/10.1530/ EC-19-0274)

Jiang M, Fu X, Yang H, Long F \& Chen J 2017 mTORC1 signaling promotes limb bud cell growth and chondrogenesis. Journal of Cellular Biochemistry 118 748-753. (https://doi.org/10.1002/jcb.25728)

Kember NF 1960 Cell division in endochondral ossification. A study of cell proliferation in rat bones by the method of tritiated thymidine autoradiography. Journal of Bone and Joint Surgery 42B 824-839. (https://doi.org/10.1302/0301-620X.42B4.824)

Kember NF 1971 Cell population kinetics of bone growth: the first ten years of autoradiographic studies with tritiated thymidine. Clinical Orthopaedics and Related Research 76 213-230. (https://doi. org/10.1097/00003086-197105000-00029)

Kember NF \& Lambert BE 1981 Slowly cycling cells in growing bone. Cell and Tissue Kinetics 14 327-330. (https://doi. org/10.1111/j.1365-2184.1981.tb00536.x)

Kobayashi T, Soegiarto DW, Yang Y, Lanske B, Schipani E, McMahon AP \& Kronenberg HM 2005 Indian hedgehog stimulates periarticular chondrocyte differentiation to regulate growth plate length independently of PTHrP. Journal of Clinical Investigation $\mathbf{1 1 5}$ 1734-1742. (https://doi.org/10.1172/JCI24397)

Kronenberg HM 2003 Developmental regulation of the growth plate. Nature 423 332-336. (https://doi.org/10.1038/nature01657)

Kronenberg HM, Karaplis AC \& Lanske B 1996 Role of parathyroid hormone-related protein in skeletal development. Annals of the New York Academy of Sciences 785 119-123. (https://doi. org/10.1111/j.1749-6632.1996.tb56249.x)

Lanske B, Amling M, Neff L, Guiducci J, Baron R \& Kronenberg HM 1999 Ablation of the PTHrP gene or the PTH/PTHrP receptor gene leads to distinct abnormalities in bone development. Journal of Clinical Investigation 104 399-407. (https://doi.org/10.1172/JCI6629)

Li Y, Trivedi V, Truong TV, Koos DS, Lansford R, Chuong CM, Warburton D, Moats RA \& Fraser SE 2015 Dynamic imaging of the growth plate cartilage reveals multiple contributors to skeletal morphogenesis. Nature Communications 6 6798. (https://doi. org/10.1038/ncomms7798)

Livet J, Weissman TA, Kang H, Draft RW, Lu J, Bennis RA, Sanes JR \& Lichtman JW 2007 Transgenic strategies for combinatorial expression of fluorescent proteins in the nervous system. Nature $\mathbf{4 5 0} 56-62$. (https://doi.org/10.1038/nature06293)

Long F, Chung UI, Ohba S, McMahon J, Kronenberg HM \& McMahon AP $2004 \mathrm{Ihh}$ signaling is directly required for the osteoblast lineage in the endochondral skeleton. Development 131 1309-1318. (https://doi. org/10.1242/dev.01006)

Lui JC 2017 Regulation of body growth by microRNAs. Molecular and Cellular Endocrinology 456 2-8. (https://doi.org/10.1016/j. mce.2016.10.024)

Lui JC, Andrade AC, Forcinito P, Hegde A, Chen W, Baron J \& Nilsson O 2010 Spatial and temporal regulation of gene expression in the mammalian growth plate. Bone 46 1380-1390. (https://doi. org/10.1016/j.bone.2010.01.373)

Lui JC, Nilsson O \& Baron J 2011 Growth plate senescence and catch-up growth. Endocrine Development 21 23-29. (https://doi. org/10.1159/000328117)

Lui JC, Jee YH, Garrison P, Iben JR, Yue S, Ad M, Nguyen Q, Kikani B, Wakabayashi Y \& Baron J 2018 Differential aging of growth plate cartilage underlies differences in bone length and thus helps determine skeletal proportions. PLoS Biology 16 e2005263. (https:// doi.org/10.1371/journal.pbio.2005263)

Mizuhashi K, Ono W, Matsushita Y, Sakagami N, Takahashi A, Saunders TL, Nagasawa T, Kronenberg HM \& Ono N 2018 Resting zone of the growth plate houses a unique class of skeletal stem cells. Nature 563 254-258. (https://doi.org/10.1038/s41586-018-0662-5)

Morishima A, Grumbach MM, Simpson ER, Fisher C \& Qin K 1995 Aromatase deficiency in male and female siblings caused by a novel mutation and the physiological role of estrogens. Journal of Clinical Endocrinology and Metabolism 80 3689-3698. (https://doi.org/10.1210/ jcem.80.12.8530621)

Morrison SJ \& Spradling AC 2008 Stem cells and niches: mechanisms that promote stem cell maintenance throughout life. Cell 132 598-611. (https://doi.org/10.1016/j.cell.2008.01.038)

Newton PT, Vuppalapati KK, Bouderlique T \& Chagin AS 2015 Pharmacological inhibition of lysosomes activates the MTORC1 signaling pathway in chondrocytes in an autophagy-independent manner. Autophagy 11 1594-1607. (https://doi.org/10.1080/1554862 7.2015.1068489)

Newton PT, Xie M, Medvedeva EV, Savendahl L \& Chagin AS 2018 Activation of mTORC1 in chondrocytes does not affect proliferation or differentiation, but causes the resting zone of the growth plate to become disordered. Bone Reports 8 64-71. (https://doi.org/10.1016/j. bonr.2018.02.006)

Newton PT, Li L, Zhou B, Schweingruber C, Hovorakova M, Xie M, Sun X, Sandhow L, Artemov AV, Ivashkin E, et al. 2019 A radical switch in clonality reveals a stem cell niche in the epiphyseal growth plate. Nature 567 234-238. (https://doi.org/10.1038/s41586-019-0989-6)

Nilsson O, Marino R, De Luca F, Phillip M \& Baron J 2005 Endocrine regulation of the growth plate. Hormone Research 64 157-165. (https://doi.org/10.1159/000088791)

Nilsson O, Weise M, Landman EB, Meyers JL, Barnes KM \& Baron J 2014 Evidence that estrogen hastens epiphyseal fusion and cessation of longitudinal bone growth by irreversibly depleting the number of resting zone progenitor cells in female rabbits. Endocrinology 155 2892-2899. (https://doi.org/10.1210/en.2013-2175)

Ohlsson C, Nilsson A, Isaksson O \& Lindahl A 1992 Growth hormone induces multiplication of the slowly cycling germinal cells of the rat https://joe.bioscientifica.com https://doi.org/10.1530/JOE-20-0045 (c) 2020 Society for Endocrinology Published by Bioscientifica Ltd. Printed in Great Britain 
tibial growth plate. PNAS 89 9826-9830. (https://doi.org/10.1073/ pnas.89.20.9826)

Ovchinnikov DA, Deng JM, Ogunrinu G \& Behringer RR 2000 Col2a1-directed expression of Cre recombinase in differentiating chondrocytes in transgenic mice. Genesis 26 145-146. (https:// doi.org/10.1002/(SICI)1526-968X(200002)26:2<145::AIDGENE14>3.0.CO;2-C)

Phornphutkul C, Wu KY, Auyeung V, Chen Q \& Gruppuso PA 2008 MTOR signaling contributes to chondrocyte differentiation. Developmental Dynamics 237 702-712. (https://doi.org/10.1002/ dvdy.21464)

Sakamoto A, Chen M, Kobayashi T, Kronenberg HM \& Weinstein LS 2005 Chondrocyte-specific knockout of the G protein $\mathrm{G}$ (s)alpha leads to epiphyseal and growth plate abnormalities and ectopic chondrocyte formation. Journal of Bone and Mineral Research 20 663-671. (https:// doi.org/10.1359/JBMR.041210)

Schipani E, Lanske B, Hunzelman J, Luz A, Kovacs CS, Lee K, Pirro A, Kronenberg HM \& Juppner H 1997 Targeted expression of constitutively active receptors for parathyroid hormone and parathyroid hormone-related peptide delays endochondral bone formation and rescues mice that lack parathyroid hormonerelated peptide. PNAS 94 13689-13694. (https://doi.org/10.1073/ pnas.94.25.13689)

Schrier L, Ferns SP, Barnes KM, Emons JA, Newman EI, Nilsson O \& Baron J 2006 Depletion of resting zone chondrocytes during growth plate senescence. Journal of Endocrinology 189 27-36. (https://doi. org/10.1677/joe.1.06489)

Smith EP, Boyd J, Frank GR, Takahashi H, Cohen RM, Specker B, Williams TC, Lubahn DB \& Korach KS 1994 Estrogen resistance caused by a mutation in the estrogen-receptor gene in a man. New England Journal of Medicine 331 1056-1061. (https://doi.org/10.1056/ NEJM199410203311604)

Smith EP, Specker B, Bachrach BE, Kimbro KS, Li XJ, Young MF, Fedarko NS, Abuzzahab MJ, Frank GR, Cohen RM, et al. 2008 Impact on bone of an estrogen receptor-alpha gene loss of function mutation. Journal of Clinical Endocrinology and Metabolism 93 3088-3096. (https://doi.org/10.1210/jc.2007-2397)

Stadtfeld M \& Graf T 2005 Assessing the role of hematopoietic plasticity for endothelial and hepatocyte development by non-invasive lineage tracing. Development 132 203-213. (https://doi.org/10.1242/ dev.01558)

St-Jacques B, Hammerschmidt M \& McMahon AP 1999 Indian hedgehog signaling regulates proliferation and differentiation of chondrocytes and is essential for bone formation. Genes and Development $\mathbf{1 3}$ 2072-2086. (https://doi.org/10.1101/gad.13.16.2072)

Usami Y, Gunawardena AT, Francois NB, Otsuru S, Takano H, Hirose K, Matsuoka M, Suzuki A, Huang J, Qin L, et al. 2019 Possible contribution of Wnt-responsive chondroprogenitors to the postnatal murine growth plate. Journal of Bone and Mineral Research 34 964-974. (https://doi.org/10.1002/jbmr.3658)

Voehringer D, Liang HE \& Locksley RM 2008 Homeostasis and effector function of lymphopenia-induced 'memory-like' T cells in constitutively T cell-depleted mice. Journal of Immunology 180 4742-4753. (https://doi.org/10.4049/jimmunol.180.7.4742)

Walker KV \& Kember NF 1972 Cell kinetics of growth cartilage in the rat tibia. I. Measurements in young male rats. Cell and Tissue Kinetics 5 401-408. (https://doi.org/10.1111/j.1365-2184.1972.tb00378.x)

Wang Y, Middleton F, Horton JA, Reichel L, Farnum CE \& Damron TA 2004 Microarray analysis of proliferative and hypertrophic growth plate zones identifies differentiation markers and signal pathways. Bone 35 1273-1293. (https://doi.org/10.1016/j.bone.2004.09.009)

Weinstein LS, Gejman PV, de Mazancourt P, American N \& Spiegel AM 1992 A heterozygous 4-bp deletion mutation in the Gs alpha gene (GNAS1) in a patient with Albright hereditary osteodystrophy. Genomics 13 1319-1321. (https://doi.org/10.1016/08887543(92)90056-x)

Yang G, Zhu L, Hou N, Lan Y, Wu XM, Zhou B, Teng Y \& Yang X 2014a Osteogenic fate of hypertrophic chondrocytes. Cell Research $\mathbf{2 4}$ 1266-1269. (https://doi.org/10.1038/cr.2014.111)

Yang L, Tsang KY, Tang HC, Chan D \& Cheah KS 2014b Hypertrophic chondrocytes can become osteoblasts and osteocytes in endochondral bone formation. PNAS 111 12097-12102. (https://doi.org/10.1073/ pnas.1302703111)

Zhou X, von der Mark K, Henry S, Norton W, Adams H \& de Crombrugghe B 2014 Chondrocytes transdifferentiate into osteoblasts in endochondral bone during development, postnatal growth and fracture healing in mice. PLoS Genetics 10 e1004820. (https://doi. org/10.1371/journal.pgen.1004820)

Received in final form 24 March 2020

Accepted 2 April 2020

Accepted Manuscript published online 2 April 2020 (c) 2020 Society for Endocrinology Published by Bioscientifica Ltd. Printed in Great Britain 